3-15-2011

\title{
Ultrasensitive Biosensing on the Zepto-Molar Level
}

\author{
Yongki Choi \\ City University of New York
}

Siu-Tung Yau

Cleveland State University, s.yau@csuohio.edu

Follow this and additional works at: https://engagedscholarship.csuohio.edu/enece_facpub

Part of the Electrical and Computer Engineering Commons

How does access to this work benefit you? Let us know!

\section{Publisher's Statement}

NOTICE: this is the author's version of a work that was accepted for publication in Biosensors \& Bioelectronics. Changes resulting from the publishing process, such as peer review, editing, corrections, structural formatting, and other quality control mechanisms may not be reflected in this document. Changes may have been made to this work since it was submitted for publication. A definitive version was subsequently published in Biosensors \& Bioelectronics, 26, 7, (03-15-2011); 10.1016/j.bios.2011.01.009

\section{Original Citation}

Choi, Y., , \& Yau, S. (2011). Ultrasensitive biosensing on the zepto-molar level. Biosensors and Bioelectronics, 26(7), 3386-3390. doi:10.1016/j.bios.2011.01.009

\section{Repository Citation}

Choi, Yongki and Yau, Siu-Tung, "Ultrasensitive Biosensing on the Zepto-Molar Level" (2011). Electrical Engineering \& Computer Science Faculty Publications. 54.

https://engagedscholarship.csuohio.edu/enece_facpub/54

This Article is brought to you for free and open access by the Electrical Engineering \& Computer Science Department at EngagedScholarship@CSU. It has been accepted for inclusion in Electrical Engineering \& Computer Science Faculty Publications by an authorized administrator of EngagedScholarship@CSU. For more information, please contact library.es@csuohio.edu. 


\title{
Ultrasensitive biosensing on the zepto-molar level
}

\author{
Yongki Choi ${ }^{\mathrm{a}, \mathrm{b}}$, Siu-Tung Yau ${ }^{\mathrm{a}, \mathrm{c}, *}$ \\ ${ }^{2}$ Department of Electrical and Computer Engineering, Cleveland State University, Cleveland, $\mathrm{OH} 44115$, USA \\ ${ }^{b}$ Department of Physics, The City University of New York, NY 10016, USA \\ 'The Applied Bioengineering Program, Cleveland State University, Cleveland, OH 44115, USA
}

\section{Introduction}

Ultrasensitive detection of substances is a current technological thrust. The demand for techniques that provide detection of extremely small quantities of substance exists in biomedicine, biological research, and environment protection (Georganopoulou et al., 2005; Nie and Emory, 1997; Yu et al., 2004). The treatments of many serious diseases will experience unprecedented development if the diseases can be detected in their early stage of development (Shipp, 2006). In addition to being ultrasensitive, the techniques should also provide analyte selectivity. In this regard, bio-electrochemical sensing appears to be a suitable approach. Electrochemical detection using enzymes as sensing elements provides substance selectivity because of the specific interaction between the enzyme and its analyte. However, the inherent low level of interfacial charge transfer due to the embedment of enzymes' active sites by protein environment creates a fundamental limit to the sensitivity of this sensing approach.

Ultrasensitive bio-detection techniques have been demonstrated previously. Glucose at 2 femto-molar $\left(10^{-15} \mathrm{M}\right)$ was detected by its electrooxidation on a carbon-platinum disk coated with glucose oxidase (GOx) and bilirubin oxidase, which were elec- trically wired to the disk with a conducting polymer (Mano and Heller, 2005). An enzyme-amplified sandwich-type amperometric assay has been used to detect a 38-base DNA strand at atto-molar $\left(10^{-18} \mathrm{M}\right)$ (Zhang et al., 2003). A capillary electrophoresis method has been used for the detection of alkaline phosphatase activity at atto-molar concentrations (Craig et al., 1996). An electrochemical immunosensor for the detection of atto-molar interferon- $\gamma$ and a nanoparticle-based bio-bar-code approach for the detect attomolar prostate-specific antigen have been reported (Dijksma et al., 2001; Nam et al., 2003).

Recently, the detection of pico-molar $\left(10^{-12} \mathrm{M}\right)$ analyte molecules using a field-effect bio-detector has been demonstrated (Choi and Yau, 2009). The detector features a voltage-controlled current amplification caused by the application of a gating voltage, which induces an interfacial electric field to modulate electron transfer between an enzyme and an electrode. This short communication reports the ultrasensitive detection of an analyte at the zepto-molar $\left(10^{-21} \mathrm{M}\right.$ or $\left.\mathrm{zM}\right)$ level with zepto-molar detection resolution, using the field-effect technique. The detection was performed with the GOx-glucose biocatalytic system and the $\mathrm{zM}$ detection was the result of optimizing the gating voltage in a higher voltage range $(\sim 0.15 \mathrm{~V})$.

\section{Material and methods}

The detection system, its operation principle and detailed information on the experiment are described in Supplementary Data. The system (see Fig. S1) consists of a conventional three-electrode 
electrochemical cell modified with an additional gating electrode for applying an external voltage $V_{\mathrm{G}}$ to the edge-plane of a highly oriented pyrolytic graphite (HOPG) working electrode, upon which an enzyme is immobilized within an area of $1 \mathrm{~mm} \times 1 \mathrm{~mm}$. A piece of copper wire coated with a thin layer of insulator was used as the gating electrode. The wire was bent to form a U-shaped structure and was attached on the working electrode next to the immobilized enzyme molecules. The enzyme immobilization method used here for GOx and ADH was described previously (Wang et al., 2006). It was shown that enzyme immobilization on the edge-plane of HOPG results in the formation of a sub-monolayer as revealed by atomic force microscopy (see Fig. S1(b) and (c)) and that the activity of the enzyme is preserved (Wang et al., 2006). Enzyme-immobilized electrodes were used as the working electrode for voltammetry measurements. A commercial $\mathrm{Ag} / \mathrm{AgCl}(3 \mathrm{M} \mathrm{KCl})$ electrode was used as the reference electrode, and a platinum wire was used as the counter electrode. The volume of the electrochemical cell was $1 \mathrm{ml}$. The cell was driven by a commercial electrochemical controller $(\mathrm{CHI}$ 660 C Work Station). A scan rate of $20-50 \mathrm{mV} / \mathrm{s}$ was used in recording voltammograms. Phosphate buffer solution (PBS) of $100 \mathrm{mM}$ at $\mathrm{pH} 7$ was used in the detection of glucose, while $100 \mathrm{mM}$ PBS at $\mathrm{pH}$ 7.8 was used in the ethanol detection. The PBS was prepared using de-ionized water (18.2 $\mathrm{M} \Omega \mathrm{cm}$ ). GOx (EC 1.1.3.4) from aspergillus niger, $\mathrm{ADH}$ (EC 1.1.1.1) from saccharomyces cerevisiae and the chemicals used in this work $(\beta-\mathrm{D}(+)$ glucose with $97 \%$ purity, ethanol with $>99.9 \%$ purity and sodium phosphate with $>99.95 \%$ purity) were purchased from Sigma-Aldrich and were used as received. All measurements were made with deaerated PBS. The detection experiment at each analyte concentration has been performed at least six times, in which identical electrodes or electrodes with minor modifications were used, and similar results were obtained.

\section{Results and discussion}

The most important property of the detector is the amplification of its signal current, which is controlled by $V_{\mathrm{G}}$. Note that $V_{\mathrm{G}}$ is not the cell potential $E$. The cyclic voltammograms (CVs) in Fig. 1(a) show the detection of glucose in the atto-molar $\left(10^{-18} \mathrm{M}\right.$ or $\left.\mathrm{aM}\right)$ range using a GOx-immobilized HOPG electrode. A series of control experiments has been performed. First, the bare HOPG electrode showed no response to glucose within the potential range used in this work, with or without $V_{\mathrm{G}}$. Also, at the aM and zM glucose levels and with $V_{\mathrm{G}}=0 \mathrm{~V}$, the exposure of the GOx-immobilized electrode to glucose produced no measurable effects on the electrode's CVs as compared to the CVs of the electrode obtained in PBS. The black CVs in Fig. 1(a) and (b) show this observation. However, when $V_{\mathrm{G}}$ was applied, the GOx-immobilized electrode showed increased currents in PBS as indicated by the green CVs in Fig. 1(a) and (b). The increased electrode currents might be caused by the electrical double layer (EDL) at the interface between the electrode and the solution. Since $V_{\mathrm{G}}$ induces negative charges on the HOPG electrode surface and positive ions at the enzyme-solution interface (Choi and Yau, 2009), the GOx molecules could have re-oriented themselves and more positive ions in the solution could have moved to the interface to cause charging current. Note that the green CV for an enzyme-immobilized electrode remains unchanged for a given $V_{\mathrm{G}}$.

The red CV in Fig. 1(a) shows the response of the GOximmobilized electrode to $70 \mathrm{aM}$ of glucose with $V_{\mathrm{G}}=0.12 \mathrm{~V}$. In the presence of $V_{G}$, the anodic currents of the CVs of the enzymeimmobilized electrode in the presence of glucose (such as the red CVs in Fig. 1(a)-(c)) are always noticeably greater than that of the green CV (the electrode's CVs in PBS). Therefore, the detection system's signal current (the oxidation current of the analyte) was obtained by subtracting the anodic current of the green CV from the corresponding anodic current of the red $\mathrm{CV}$ at a particular cell potential for different glucose concentrations. Obtained on the aM glucose level with $V_{\mathrm{G}}=0.12 \mathrm{~V}$, the electrode's glucose calibration curve, the plot showing the relation between the signal current (glucose oxidation current) and the glucose concentration, is shown in the inset of Fig. 1(a). The calibration curve shows that the lowest detectable glucose concentration is $5 \mathrm{aM}$, above which increments of 5, 10, 20 and $30 \mathrm{aM}$ in glucose concentration result in distinguishable signal current values. Note that $V_{\mathrm{G}}=0.12 \mathrm{~V}$ is the optimum value of $V_{\mathrm{G}}$ (see below) for the detection on the aM glucose level.

Detection of glucose in the zepto-molar range was obtained with $V_{\mathrm{G}}=0.15 \mathrm{~V}$ (the optimized value) as shown in Fig. 1(b). The green and red CVs and the calibration curve in the inset were obtained under similar conditions as described above. The calibration curve shows that the lowest detectable glucose concentration is $50 \mathrm{zM}$ and the detection has a detection resolution of $50 \mathrm{zM}$. The error bars show that the current of each data point distinctively represents the corresponding concentration. The experiments described above have been performed six times and reproducible results have been obtained.

The existence of an electric field at the solution-working electrode interface caused by $V_{\mathrm{G}}$ was described previously (Choi and Yau, 2009). In our experimental setup, negative charges are induced on the HOPG electrode's surface and positive ions are induced at the solution-enzyme interface, therefore establishing the field within the enzyme (Supplementary Data). This field reduces the effective height of the tunnel barrier (the polypeptide networks) between the enzyme's active site and the electrode and enhances the tunneling rate (Tans et al., 1998), resulting in amplified signal current.

In order to show the extended applicability of the detection technique, we have applied this technique to the ADH-ethanol system. Fig. 1(c) shows the detection of ethanol using the ADHimmobilized electrode in the femto-molar $\left(10^{-15} \mathrm{M}\right.$ or $\left.\mathrm{fM}\right)$ range achieved with $V_{\mathrm{G}}=0.15 \mathrm{~V}$ (the optimized value). The green and red CVs and the calibration curve carry similar information as described above. The calibration curve indicates that the lowest detectable ethanol concentration is $10 \mathrm{fM}$, above which increments of $25 \mathrm{fM}$ in ethanol concentration result in distinguishable current values. Fig. 1 shows that, by applying $V_{\mathrm{G}}$, the current level of the detection system for atto and zepto-molar ranges of analyte concentration can be controlled in the nano-ampere range for convenient electronic signal processing.

Two interesting effects have been observed in the experiment. The first effect is that, although the electric field did not produce permanent detrimental effect on the activities of the enzymes, however, when the field was high enough, it produced a temporary reduction in the signal current. In Fig. 2(a), the glucose oxidation current (the signal current) of a GOx-immobilized electrode is plotted versus $V_{\mathrm{G}}$ for $30 \mathrm{aM}$ glucose. The plot shows that initially the current increases with increasing $V_{\mathrm{G}}$, indicating the amplification of the signal current. The current reaches a maximum value when $V_{\mathrm{G}}$ reaches a critical value $V_{\mathrm{C}}\left(V_{\mathrm{C}}=0.12 \mathrm{~V}\right.$ in the present case), after which the current decreases with further increase in $V_{G}$. When $V_{G}$ is reversed, the current follows almost the same path to the initial value as indicated by the arrows. This effect suggests that a certain amount of the GOx molecules on the electrode was temporarily "disabled" (reduced enzymatic activity) by the field due to a certain mechanism, which occurred when the field became high enough. The second effect is related to the saturation of the signal current in the calibration curves in Fig. 1(a) and (b). The calibration curves show current saturation due to the Michaelis-Menten kinetics. However, the atto-molar calibration curve in Fig. 1(a) suggests that saturation should not occur in the zM range. Here, we propose a possible mechanism for the first effect. It will become obvious that the second effect could be the result of the first effect.

Since the enzymes carry surface charges (Hecht et al., 1993; Sund and Theorell, 1963), the interfacial field could have re- 

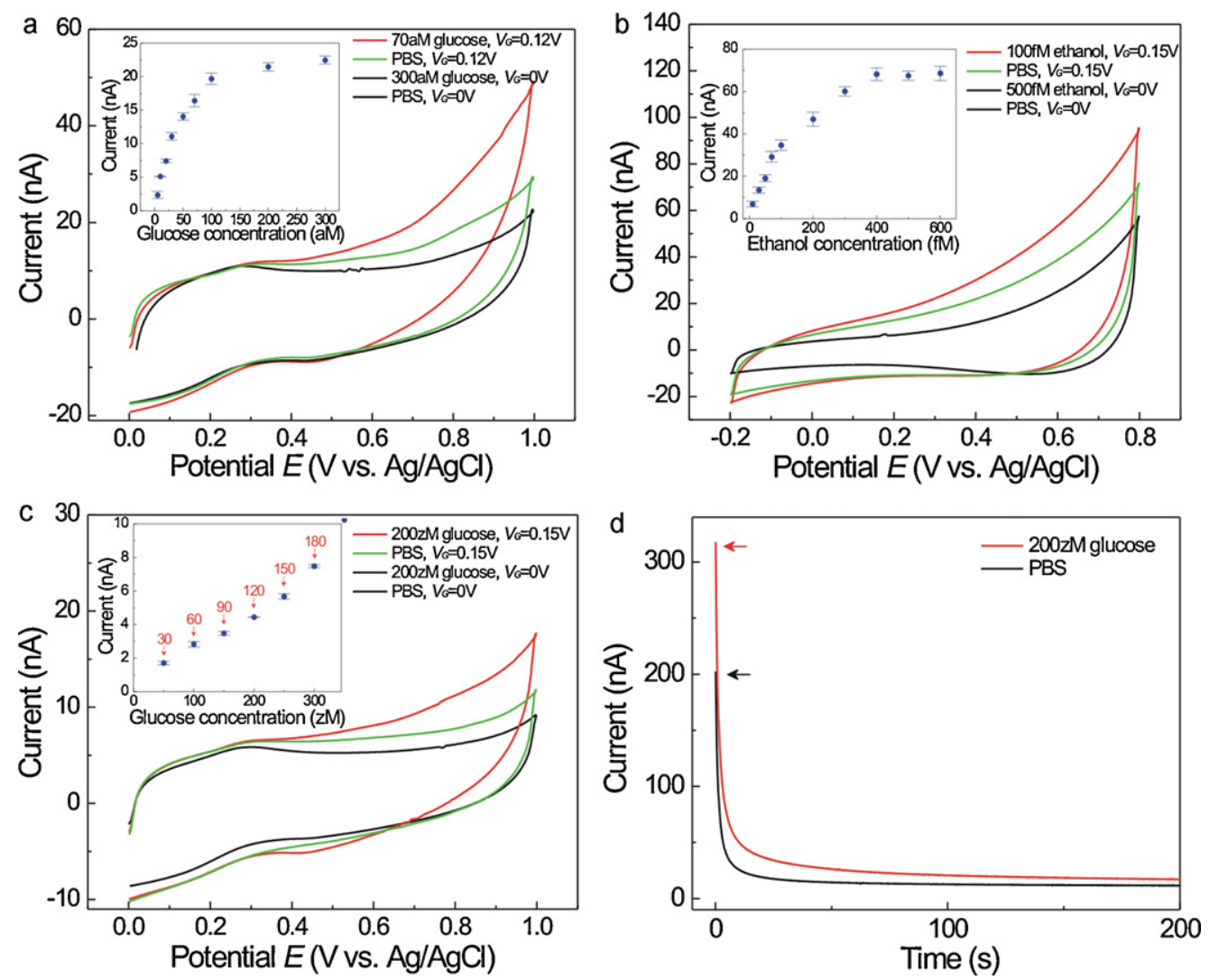

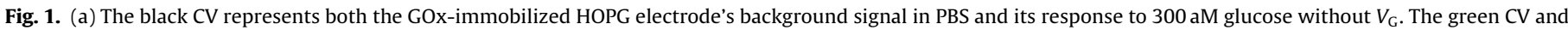

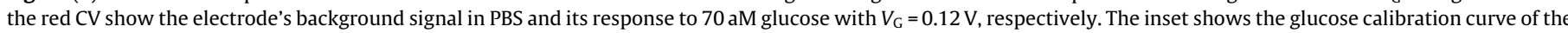

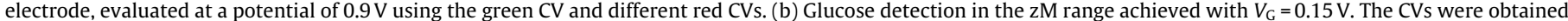

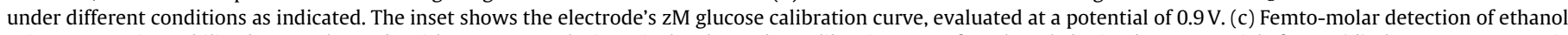

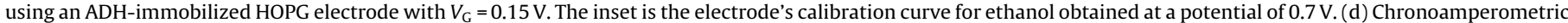

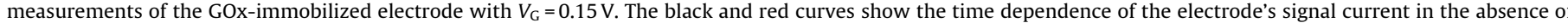

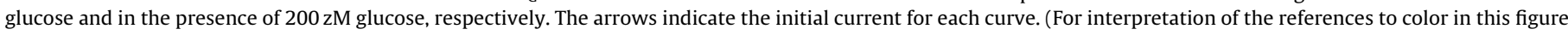
legend, the reader is referred to the web version of the article.)

oriented them on the electrode (Kranich et al., 2008; Strauss et al., 2004; Willit and Bowden, 1987) so that they were temporarily "disabled" in carrying out their enzymatic activities, the result of which being diminished catalytic activity or interfacial tunneling or both. In the case of GOx, the enzyme has an isoelectric point of $\mathrm{pH} 4.2$ and, therefore, it carries a net negative charge at neutral pH (Courjean et al., 2010). This charge may cause GOx to re-orient itself on the electrode in the presence of an interfacial field. Re-orientation of GOx may also be caused by local charge residues on GOx. It is known that both positively charged arginine residues and negatively charged aspartic acid residues are located near the opening of the active channel, which houses the active site of GOx. The re-orientation may cause blockage of the channel by the electrode or by neighboring GOx molecules to prevent glucose
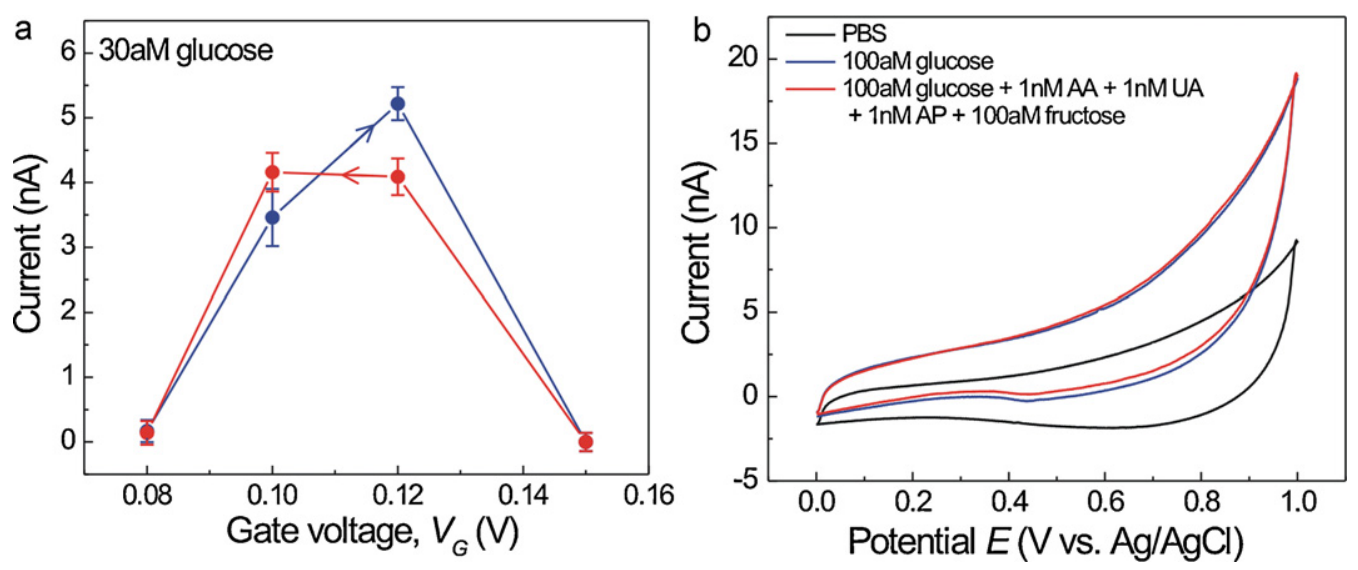

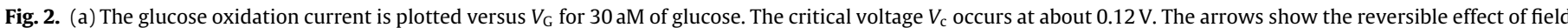

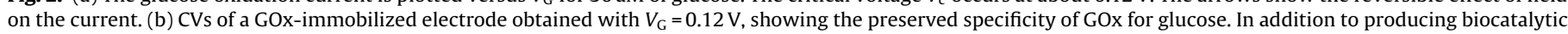

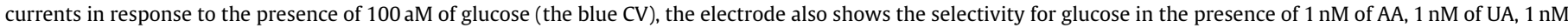
of AP and $100 \mathrm{aM}$ of fructose (the red CV). (For interpretation of the references to color in this figure legend, the reader is referred to the web version of the article.) 
from reaching the active site, resulting in the "disable" scenario. When the field is turned off, the enzyme molecules may return to their original orientations. In fact, such field-induced reversible reorientation of redox proteins with respect to electrodes has caused changes in redox currents as observed previously (Kranich et al., 2008; Strauss et al., 2004; Willit and Bowden, 1987). In the present work, when $V_{\mathrm{G}}$ was increased beyond $V_{C}$, the field became strong enough to "disable" a certain amount of enzyme molecules and the number of functioning enzyme molecules on the electrode were reduced, causing the observed saturation effect in the $\mathrm{zM}$ range.

It was observed that the selectivity of GOx for glucose in the presence of the electric field was retained even for extremely low glucose concentrations. Fig. 2(b) shows that, with the field produced by $V_{\mathrm{G}}=0.12 \mathrm{~V}$, the response of a GOx-immobilized electrode to $100 \mathrm{aM}$ of glucose (the blue $\mathrm{CV}$ ) is almost indistinguishable from the electrode's response (the red CV) to $100 \mathrm{aM}$ of glucose in the presence of $1 \mathrm{nM}$ of ascorbic acid (AA), $1 \mathrm{nM}$ of 4-acetamidophenol (AP), $1 \mathrm{nM}$ of uric acid (UA) and $100 \mathrm{aM}$ fructose, which are interfering substances in the body fluid. Thus, the substance selectivity of GOx (enzyme's specificity for its analyte) has not been affected by the electric field in the presence of interfering substances, whose concentration are $10^{7}$ times higher than that of glucose (the analyte). Note that at the physiological level, the ratio of these interfering substances to glucose is less than unity (Christison and MacKenzie, 1993; Ernst et al., 2002).

The calibration curves in Figs. 1(a) and (b) clearly indicate that, in the atto- and zepto-molar concentration ranges, the signal current changes as the concentration is changed. In the inset of Fig. 1(b), each data point is associated with the number of glucose molecules in the cell. As shown, the system was able to detect a minimum of 30 glucose molecules present in the cell and showed response to each incremental change in units of 30 glucose molecules in the cell. The total charge transferred $Q=n F N$, where $n=2, F$ the Faradaic constant, and $N$ the number of mole electrolyzed, is estimated to be $10^{-17} \mathrm{C}$ produced by 30 glucose molecules so that the current is estimated to be 2.5 atto-ampere, using a time interval of 0.4 s during which the anodic current increases noticeably as a result of scanning the cell potential from 0.8 V to 1.0 V (Bard and Faulkner, 2001). However, the detection current observed in zepto-molar range is on the nano-ampere level. Several possible processes that cause the phenomenon are given below.

The high interfacial field $\left(\sim 10^{7} \mathrm{~V} / \mathrm{cm}\right)$ may cause a complex situation for the glucose oxidation reaction. First, the enzyme's active site and the electrode can be treated as electron donor and acceptor, respectively (Marcus and Sutin, 1985). The high field causes large downward distortion of the barrier-height $\Phi_{\mathrm{B}}$ (Snow et al., 1998 ) between the active site and the electrode so that the electron transfer rate $k_{\text {et }} \propto \exp \left(-\Phi_{\mathrm{B}}{ }^{1 / 2} d\right)$, where $d$ is separation between the active center and the electrode, becomes large due to the nonlinear dependence (Page et al., 1999). On being oxidized, glucose is instantaneously converted to glucono-lactone. Since FAD, the active center of GOx, is also a cofactor for oxidation of gluconolactone (Salusjarvi et al., 2004) and it is readily available to carry out the oxidation of glucono-lactone, more current will be produced. A second possible scenario involves stripping electrons directly from glucose or from glucono-lactone. Because of the highly distorted tunnel barrier, the conductance between the active site and the electrode becomes enhanced so that the active site is effectively electrically connected to the electrode. Therefore, the active site and the electrode form a complex, which is capable of stripping electrons from glucose (Kokoh et al., 1992; Park et al., 2003) Previous observation shows that 18 electrons can be extracted (Tominaga et al., 2005). In this case, the GOx acts as a bridge (Tominaga et al., 2005) between glucose and the electrode. These two possible processes may individually or collectively contribute

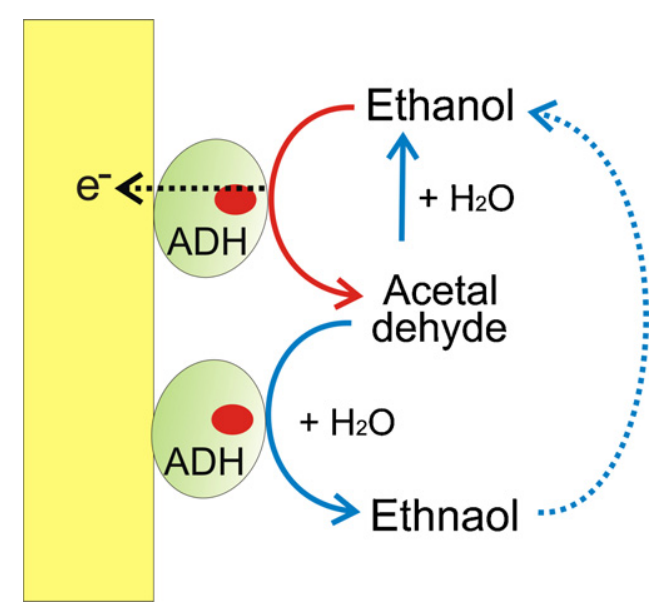

Fig. 3. The cyclic oxidation and conversion of ethanol. In an ADH-ethanol complex, ethanol is oxidized to acetaldehyde. In some part of ADH and unoccupied $\mathrm{ADH}$, acetaldehyde can be reduced to ethanol by a streochemically cryptic reduction reaction of $\mathrm{ADH}$

the observed enhanced oxidation current. Fig. 1(d) shows the time dependences of the electrode's signal current with $V_{\mathrm{G}}=0.15 \mathrm{~V}$ in the absence of glucose (the black curve) and presence of $200 \mathrm{zM}$ glucose (the red curve). At $t=0$, the slower current decay in the red curve reflects the oxidation of glucose in the sample. In fact, the red curve decays much slower than the black curve during the period of the first $40 \mathrm{~s}$ used to record the CVs. This observation may be due to the continuous oxidation of the limited number of glucose molecules after their enzyme-catalyzed oxidation has taken place.

The ethanol detection current in the femto-molar range is also on the nano-ampere level. Similarly, the field-induced ethanol oxidation reaction may also involve stripping electrons directly from ethanol as explained above. Previous observation shows that 12 electrons can be extracted from ethanol (Lamy and Belgsir, 2003). A cyclic reaction of ethanol is suggested as shown in Fig. 3. In an ADH-ethanol complex, ethanol is oxidized to acetaldehyde. Since the electrode contains both ethanol-bound $\mathrm{ADH}$ and ethanol-free unbounded $\mathrm{ADH}$, acetaldehyde could be reduced to ethanol by a streochemically cryptic reduction reaction of ADH as shown in Fig. 3 (Maconi et al., 1988). Therefore, the cyclic conversion between ethanol and acetaldehyde may occur, which produces enhanced oxidation current. These possible processes may individually or collectively cause the observed enhanced oxidation current. Note that in order to participate in the possible processes presented above, an analyte molecule must have already diffused to the enzymeimmobilized electrode to participate in the bio-catalysis. While these processes take place, mass transport of other molecules could be in progress.

\section{Conclusions}

We showed unequivocally the bio-detection of glucose in the zepto-molar range with the lowest detectable glucose concentration of $50 \mathrm{zM}$, which is equivalent to detecting 30 glucose molecules, and a detection resolution of $50 \mathrm{zM}$ glucose. The detection was achieved by applying a gating voltage to the sensing electrode, which resulted in the amplification of the biocatalytic current. The increased biocatalytic current is believed to be the result of the modification of the tunnel barrier at the enzyme-electrode interface by the interfacial electric field due to the gating voltage. Using the GOx-glucose system, we showed that the substance selectivity of the enzyme has not been compromised by the field. We presented three possible processes for the observed signal current magnitudes, although the exact mechanism remains 
to be determined. Further investigation will be performed to elucidate the current amplification effect.

\section{Acknowledgements}

This work was supported by American Diabetes Association (grant number 7-08-RA-191) and Cleveland State University (Research Challenge Award).

\section{References}

Bard, A.J., Faulkner, L.R., 2001. Electrochemical Methods, 2nd ed. John Wiley \& Sons, New Jersey.

Choi, Y., Yau, S.T., 2009. Anal. Chem. 81, 7123-7126.

Christison, G.B., MacKenzie, H.A., 1993. Med. Boil. Eng. Comp. 31, 284-290.

Courjean, O., Flexer, V., Prevoteau, A., Suraniti, E., Mano, N., 2010. Chem. Phys. Chem $11,2795-2797$

Craig, D.B., Wong, J.C.Y., Dovichi, N.J., 1996. Anal. Chem. 68, 697-700.

Dijksma, M., Kamp, B., Hoogvliet, J.C., Bennekom, W.P.v., 2001. Anal. Chem. 73 , 901-907.

Ernst, H., Rob, B., Knoll, M., 2002. Anal. Bioanal. Chem. 373, 758-761.
Georganopoulou, D.G., Chang, L., Nam, J.M., Thaxton, C.S., Mufson, E.J., Klein, W.L Mirkin, C.A., 2005. Proc. Natl. Acad. Sci. U.S.A. 102, 2273-2276.

Hecht, H.J., Kalisz, H.M., Hendle, J., Schmid, R.D., Schomburg, D., 1993. J. Mol. Biol. 229, 153-172.

Kokoh, K.B., Leger, J.M., Beden, B., Lamy, C., 1992. Electrochim. Acta 37, 1333-1342. Kranich, A., Ly, H.K., Hildebrandt, P., Murgida, D.H., 2008. J. Am. Chem. Soc. 130 9844-9848.

Lamy, C., Belgsir, E.M., 2003. Technology and Applications. John Wiles \& Sons.

Maconi, E., Griffini, A., Cavazzoni, V., Aragozzini, F., 1988. Biochem. J. 250, 929-932. Mano, N., Heller, A., 2005. Anal. Chem. 77, 729-732.

Marcus, R.A., Sutin, N., 1985. Biochim. Biophys. Acta 811, 265-322.

Nam, J.-M., Thaxton, C.S., Mirkin, C.A., 2003. Science 301, 1884-1886.

Nie, S., Emory, S.R., 1997. Science 275, 1102-1106.

Page, C.C., Moser, C.C., Chen, X., Dutton, P.L., 1999. Nature 402, 47-52.

Park, S., Chung, T.D., Kim, H.C., 2003. Anal. Chem. 75, 3046-3049.

Salusjarvi, T., Kalkkinen, N., Miasnikov, A.N., 2004. Appl. Environ. Microbiol. 70 5503-5510.

Shipp, G., 2006. Biotech. Healthcare, 35-40.

Snow, E.S., Campbell, P.M., Rendell, R.W., Buot, F.A., Park, D., Marrian, C.R.K., Magno, R., 1998. Appl. Phys. Lett. 72, 3071.

Strauss, E., Thomas, B., Yau, S.T., 2004. Langmuir 20, 8768-8772.

Sund, H., Theorell, H., 1963. In: Boyer, P., Nardy, B., Myrback, K. (Eds.), The Enzymes., 2nd ed. Academic Press, New York.

Tans, S.J., Verschueren, A.R.M., Dekker, C., 1998. Nature 393, 49-52.

Tominaga, M., Shimazoe, T., Nagashima, M., Taniguchi, I., 2005. Electrochem. Commun. 7, 189-193.

Wang, G., Thai, N.M., Yau, S.-T., 2006. Electrochem. Commun. 8, 987-992.

Willit, J.L., Bowden, E.F., 1987. J. Electroanal. Chem. 221, 265-274.

Yu, F., Persson, B., Lofas, S., Knoll, W., 2004. J. Am. Chem. Soc. 126, 8902-8903.

Zhang, Y., Kim, H.-H., Heller, A., 2003. Anal. Chem. 75, 3267-3269. 